\title{
The effect of intramolecular processes on the polarizabilities and hyperpolarizabilities of some amines
}

\author{
J. Waite and M. G. Papadopoulos \\ Theoretical and Physical Chemistry Institute, National Hellenic Research Foundation, Vas. Constantinou 48, GR-116 35 \\ Athens, Greece
}

(Received 17 January 1984; accepted 19 October 1984)

\begin{abstract}
We demonstrate that small, properly extended and optimized basis sets, within CNDO, are appropriate for the calculation of the electric polarizabilities and hyperpolarizabilities of amines. Employing such a wave function we show that the intramolecular processes (inversion and rotation) in some aromatic amines

$\left[\mathrm{C}_{6} \mathrm{H}_{5} \mathrm{NH}_{2}, \mathrm{C}_{6} \mathrm{H}_{5} \mathrm{~N}\left(\mathrm{CH}_{3}\right)_{2}\right.$, and 1- $\left.\mathrm{C}_{10} \mathrm{H}_{7} \mathrm{NH}_{2}\right]$ have a small effect on the average polarizability and second hyperpolarizability while large changes are observed in the anisotropies of the charge cloud and the first hyperpolarizability. These observations are discussed and rationalized.
\end{abstract}

\section{INTRODUCTION}

The electric polarizability $\alpha$ and hyperpolarizabilities $\beta$ and $\gamma$ are at the heart of many important phenomena (e.g., intramolecular forces, chemical reactivity, etc.) and thus it is not surprising that they are the subject of intensive research. ${ }^{1-3}$

At the experimental level a variety of methods has been applied to measure the electric properties of large numbers of molecules. ${ }^{1.4 .5}$ Furthermore, several computational approaches have been used to study various aspects of molecular polarization. ${ }^{2,3}$ Rigorous procedures, however, have been restricted to small molecules since computational treatments, by $a b$ initio methods of the hyperpolarizabilities in particular, encounter severe difficulties. An outline of the problems that require sufficient solutions include: (a) the basis set. Large sets of functions are required by perturbation theory for the accurate description of the outer regions of the charge cloud necessary for hyperpolarizability calculations. (b) The correlation effects. Their contribution is of crucial importance for a meaningful approach to molecular polarization. $^{6-8}$ (c) The environment. The significance of its influence particularly on $\beta$ has already been noted. ${ }^{9.10}$ However, these phenomena are tacitly recognized to be computationally unmanageable, at present, and usually are ignored."

For these reasons, the qualitative difference, which exists between small molecules (say less than ten electrons) and large ones (where phenomena like delocalization, charge transfer processes, etc. can be found) constitutes a barrier which currently may be crossed only by judiciously designed semiempirical methods. Such a method, which gives due consideration to the reasonable, yet economical (in terms of computer time and storage) description of the electric polarization phenomenon, has been reported and successfully applied to a large series of hydrocarbons. ${ }^{12-14}$

In this communication we demonstrate that molecules with functional groups containing nitrogen can also be successfully described by small, extended, and properly optimized basis sets. Further, we consider the possibility of designing basis sets for complex molecules by making use of the bases of appropriately defined fragments or models of them.

The above considerations helped to define the theoretical framework required to examine the effect of an intramolecular process (e.g., inversion or rotation of the amine group) on the polarizabilities, hyperpolarizabilities, and their anisotropies. Specifically, employing aniline as a test model, we have invoked three different phenomena (geometry changes, coupling of the $\pi$ electron system/ charge transfer, and the inductive effect) to explain the observed variation of the properties with rotation and inversion. The generality of the findings in aniline has been confirmed employing $\mathrm{NH}_{3}, \mathrm{C}_{6} \mathrm{H}_{5} \mathrm{~N}\left(\mathrm{CH}_{3}\right)_{2}$, and 1$\mathrm{C}_{10} \mathrm{H}_{7} \mathrm{NH}_{2}$.

It is noted that the reported computations allow some insight into processes which are difficult to monitor by experimental procedures used to determine linearities and nonlinearities. In addition, these processes are illuminated by simultaneously correlating various electric properties, determined within the same theoretical framework.

Previously these interactions have been investigated employing energy as the probe. ${ }^{15-17}$ The energy, however, is a property most appropriate to give information about the inner parts of the charge cloud, while the electric properties are suitable for the investigation of the outer regions of the charge distribution. Thus the results reported here are complementary to previous studies of these intramolecular processes and although some of the absolute values may prove not to be definitive, at least they can serve as a starting point for more refined calculations.

\section{REVIEW}

There are very few theoretical investigations concerning hyperpolarizabilities of large molecules containing heteroatoms. This is not unexpected, however, considering 
TABLE I. The average ionization potentials $I_{\mu}$ for the vacant orbitals of $\mathrm{N}(\mathrm{eV})$. $^{\mathrm{N}}$

\begin{tabular}{ccl}
\hline \hline Atom & Orbital & $I_{\mu}$ \\
\hline \multirow{2}{*}{$\mathrm{N}$} & $3 s$ & 4.328 \\
& $3 p$ & 3.146 \\
& $3 d$ & 2.456 \\
& $4 s$ & 2.582 \\
& $4 p$ & 2.201 \\
& $4 d$ & 1.782 \\
& $4 f$ & 0.85 \\
\hline \hline
\end{tabular}

- Reference 49.

that severe difficulties are encountered if one wants calculated values that have a small error, compared to results which are considered reliable.

For compounds containing heteroatoms we note the theoretical studies of hyperpolarizabilities by (a) Hameka and his co-workers, ${ }^{18}$ who employed a Hückel SCF wave function to calculate $\gamma$ of nitrogen containing heterocycles and nitriles. (b) Zyss, ${ }^{19-21}$ who reported INDO-FPT computations for $\beta$ of some substituted benzenes. This author has applied the same method to compute $\gamma$ of nitrobenzene. ${ }^{20}$ Further, Zyss and Berthier ${ }^{22}$ have studied urea employing INDO-FPT and, at the $a b$ initio level, a Hartree-Fock-FPT scheme. (c) Lalama and Garito have used a CNDO approach to compute the first hyperpolarizability of aniline, nitrobenzene, and $p$-nitroaniline. ${ }^{23}$

Finally, one should mention the problems related to the comparison of theoretically and experimentally deter- mined electric properties. First, there is uncertainty concerning the choice of the right local field model by which the macroscopic third order nonlinearity is related to the microscopic value. ${ }^{9}$ Second, $\beta$ is strongly dependent on environmental effects (intermolecular forces, intermolecular orientational, or electronic correlations, etc.). ${ }^{9,10,24-25}$

Due to these problems, the $\beta$ values reported here are not, in general, directly comparable with values determined through experiment. However the computed $\beta$ values provide a measure of the first hyperpolarizability of the molecule in the absence of any intermolecular interactions. This allows a direct estimation to be made of the contributions, from such interactions, to $\beta$ and thus helps in the establishment of a general and reliable model for determining local field effects.

\section{DEFINITIONS}

The induced moment of a molecule in a static electric field $F$ is given by ${ }^{26}$

$$
\mu_{\alpha}=\alpha_{\alpha \beta} F_{\beta}+\frac{1}{2} \beta_{\alpha \beta \gamma} F_{\beta} F_{\gamma}+\frac{1}{6} \gamma_{\alpha \beta \gamma \delta} F_{\beta} F_{\gamma} F_{\delta}+\cdots,
$$

where $\mu_{\alpha}$ is the $\alpha$ component of the induced dipole moment, $\alpha_{\alpha \beta}$ is the $\alpha \beta$ component of the polarizability, $\beta_{\alpha \beta \gamma}$ is the $\alpha \beta \gamma$ component of the first hyperpolarizability, $\gamma_{\alpha \beta \gamma \delta}$ is the $\alpha \beta \gamma \delta$ component of second hyperpolarizability, and $\alpha, \beta, \gamma, \delta$, the Greek suffixes, denote Cartesian components.

The average values of the polarizability and the hyperpolarizabilities, which are of interest in this work, $\operatorname{are}^{26}$

TABLE II. Design of basis sets for aniline."

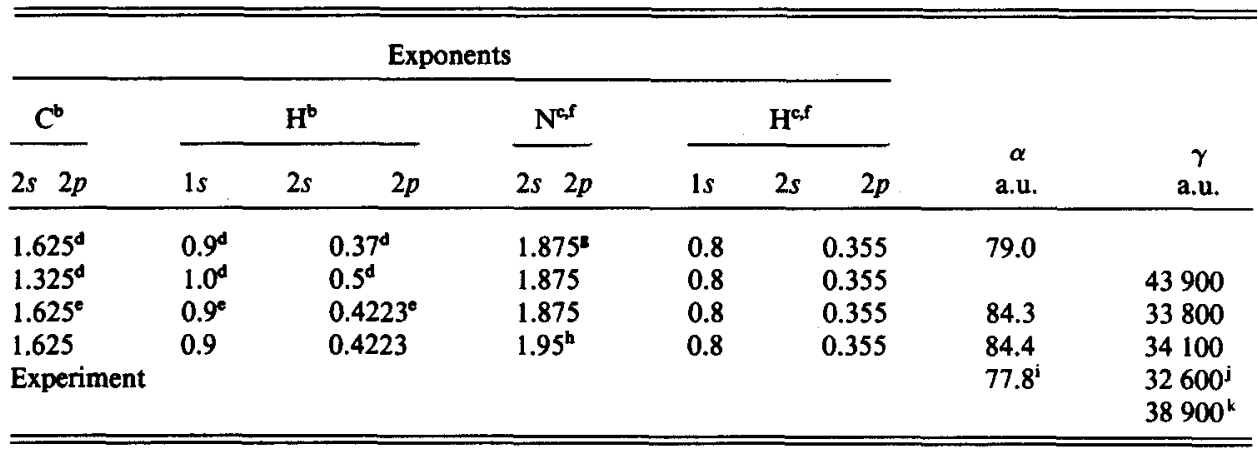

- Note. (i) Table XVII gives the required factors to convert the property values, which are reported in a.u. to values in esu or SI. (ii) The following convengence criteria have been used for all the reported computations herein:

and

$$
\left|{ }^{0} R_{y}^{k}-{ }^{0} R_{y}^{k-1}\right|<10^{-4} \text { for every } i j
$$

$$
\left|E^{k}-E^{k-1}\right|<10^{-10} \text { a.u., }
$$

where ${ }^{0} R_{i j}^{k}$ is the $i j$ th element of the unperturbed density matrix at the $k$ th cycle and $E^{k}$ is the corresponding electronic energy. For the properties $\left|{ }^{n} R_{i j}^{k}-{ }^{n} R_{i j}^{k-1}\right|<10^{-6} \times N$ for every $i j$ where $n=1,2$ is the order of the perturbed density matrix and $N$ is the number of orbitals (Ref. 43).

These atoms belong to the ring of $\mathrm{C}_{6} \mathrm{H}_{5} \mathrm{NH}_{2}$.

c These atoms belong to the amine group.

- These exponents have been taken from Ref. 14 .

- These exponents have been taken from Ref. 43.

f These exponents have been optimized with respect to $\alpha$ and $\gamma$ of $\mathrm{NH}_{3}$. The computed properties of $\mathrm{NH}_{3}$, employing this basis, are given in Table VI.

'This exponent is given by the Burns' rules (Ref. 44).

h This exponent is given by the Slater's rules (Ref. 45).

i Reference 41 .

j Reference 30.

Reference 42 
TABLE III. Literature survey of theoretical and experimental work reporting the polarizability and hyperpolarizability values of $\mathrm{NH}_{\mathbf{3}}$.

\begin{tabular}{|c|c|c|}
\hline Property & Theory & Experiment \\
\hline $\begin{array}{l}\alpha \\
\beta \\
\gamma\end{array}$ & $\begin{array}{r}14.7,16.1, b^{\mathrm{b}} 15.7,^{\mathrm{c}} 12.2 .^{\mathrm{d}} \\
-13.1,-15.1,-12.9,\end{array}$ & $\begin{array}{r}15.0^{\circ} \\
-48.4^{\mathrm{h}} \\
6090^{\mathrm{h}}\end{array}$ \\
\hline $\begin{array}{l}\text { Reference } 33 . \\
\text { ' Reference } 34 . \\
\text { c Reference } 35 . \\
\text { d Reference } 36 .\end{array}$ & $\begin{array}{l}\text { 'Reference } 37 . \\
\text { 'Reference } 38 . \\
\text { Reference } 39 . \\
\text { ' Reference } 40 .\end{array}$ & \\
\hline
\end{tabular}

$$
\begin{gathered}
\alpha=\frac{1}{3}\left(\alpha_{x x}+\alpha_{y y}+\alpha_{z z}\right), \\
\beta=\frac{3}{5}\left(\beta_{z x x}+\beta_{z y y}+\beta_{z z z}\right), \\
\gamma=\frac{1}{5}\left(\gamma_{x x x x}+\gamma_{y y y y}+\gamma_{z z z z}+2 \gamma_{x x y y}+2 \gamma_{x x z z}+2 \gamma_{y y z z}\right) .
\end{gathered}
$$

The charge reorganization, which is induced by the various intramolecular processes considered, is probed by changes in the values of the following anisotropy expressions:

$$
\begin{aligned}
& \zeta^{2}=\frac{1}{2}\left[\left(\alpha_{x x}-\alpha_{y y}\right)^{2}+\left(\alpha_{x x}-\alpha_{z z}\right)^{2}+\left(\alpha_{y y}-\alpha_{z z}\right)^{2}\right] \\
& \Delta \alpha=\alpha_{z z}-\frac{1}{2}\left(\alpha_{x x}+\alpha_{y y}\right), \\
& \Delta \gamma=\gamma_{z z z z}-\frac{1}{2}\left(\gamma_{x x c x}+\gamma_{y y y y}\right) .
\end{aligned}
$$

It is noted that $\zeta^{2}$ appears in the Kerr constant, while $\Delta \alpha$ is the anisotropy along the dipole moment (which is the $z$ axis) and the expression for $\Delta \gamma$ has been defined by analogy to the expression for $\Delta \alpha{ }^{27}$

\section{COMPUTATIONAL APPROACH}

\section{A. The CHF-PT-EB-CNDO method}

Our computational scheme relies on a CNDO wave function defined in terms of properly optimized and extended basis sets. ${ }^{12-14}$ This function is perturbed by employing the method developed by McWeeny and his co-workers. ${ }^{28}$ Full details are given in Refs. 12-14. The average ionization potentials $I_{\mu}$ for the atomic vacant orbitals of $N$ are given in Table $\mathrm{I}$. The convergence criteria for the computations are given in note (ii) of Table II.

\section{B. A working hypothesis}

The backbone of this conjecture is that one may build basis sets for molecules employing the orbitals of appropriately chosen fragments, or models of them. There is increasing evidence to suggest that this hypothesis is physically sound. ${ }^{29}$ The following examples are related to the objectives of this communication. Thus, we note in Table II the remarkably good results obtained for aniline by using orbitals for the amine group, optimized with respect to $\alpha$ and $\gamma$ of $\mathrm{NH}_{3}$ (Table III) while for the calibration of the ring orbitals, we have used three options according to which we have optimized the ring functions with respect to (i) the polarizability of $\mathrm{C}_{6} \mathrm{H}_{6}$; (ii) the hyperpolarizability of $\mathrm{C}_{6} \mathrm{H}_{6}$; and (iii) the polarizability and the hyperpolarizability of $\mathrm{C}_{6} \mathrm{H}_{6}$. We observe that there is good agreement between the experimental and calculated values of $\alpha$ and $\gamma$ (Table II).

Further evidence for the aptitude of the stated conjecture, to provide a viable algorithm for designing basis sets, is provided by the second hyperpolarizability of $\mathrm{C}_{6} \mathrm{H}_{5} \mathrm{~N}\left(\mathrm{CH}_{3}\right)_{2}$, since there is reasonable agreement between the experimental (51 600 a.u. ${ }^{30}$ ) and the computed (45 000 a.u.) nonlinearities.

\section{Analysis of the effect of the basis set on $\alpha, \beta$, and $\gamma$}

The choice of the appropriate basis set is essential to any computational method which aims at reasonably accurate induced moments. ${ }^{2}$ However very little is known,

\begin{tabular}{|c|c|c|c|c|c|c|c|c|c|c|c|c|c|c|c|c|c|c|}
\hline \multirow[b]{3}{*}{ No. } & \multicolumn{15}{|c|}{ Exponents } & \multirow[b]{3}{*}{$\alpha$} & \multirow[b]{3}{*}{$\beta$} & \multirow[b]{3}{*}{$\gamma$} \\
\hline & \multicolumn{6}{|c|}{$\mathbf{H}$} & \multicolumn{9}{|c|}{$\mathbf{N}$} & & & \\
\hline & $1 s$ & $2 s$ & $2 p$ & $3 s$ & $3 p$ & $3 d$ & $2 s$ & $2 p$ & $3 s$ & $3 p$ & $3 d$ & $4 s$ & $4 p$ & $4 d$ & $4 f$ & & & \\
\hline 1 & 1.2 & & & & & & 1.95 & 1.95 & & & & & & & & 2.6 & -1.6 & 11 \\
\hline 2 & 1.2 & 0.6 & & & & & 1.95 & 1.95 & & & & & & & & 3.9 & -5.8 & 34 \\
\hline 3 & 1.2 & 0.6 & 0.6 & & & & 1.95 & 1.95 & & & & & & & & 9.9 & -58.1 & 1050 \\
\hline 4 & 1.2 & 0.6 & 0.6 & & & & 1.95 & 1.95 & 0.65 & & & & & & & 10.3 & -61.3 & 1060 \\
\hline 5 & 1.2 & 0.6 & 0.6 & & & & 1.95 & 1.95 & 0.65 & 0.65 & & & & & & 13.4 & -70.0 & 1300 \\
\hline 6 & 1.2 & 0.6 & 0.6 & & & & 1.95 & 1.95 & 0.65 & 0.65 & 0.65 & & & & & 18.0 & -79.5 & 1900 \\
\hline 7 & 1.2 & 0.6 & 0.6 & 0.4 & & & 1.95 & 1.95 & 0.65 & 0.65 & 0.65 & & & & & 21.7 & -142 & 3860 \\
\hline 8 & 1.2 & 0.6 & 0.6 & 0.4 & 0.4 & & 1.95 & 1.95 & 0.65 & 0.65 & 0.65 & & & & & 36.7 & 343 & 16800 \\
\hline 9 & 1.2 & 0.6 & 0.6 & 0.4 & 0.4 & 0.4 & 1.95 & 1.95 & 0.65 & 0.65 & 0.65 & & & & & 53.0 & -415 & 28800 \\
\hline 10 & 1.2 & 0.6 & 0.6 & 0.4 & 0.4 & 0.4 & 1.95 & 1.95 & 0.65 & 0.65 & 0.65 & 0.488 & & & & 58.1 & -500 & 35000 \\
\hline 11 & 1.2 & 0.6 & 0.6 & 0.4 & 0.4 & 0.4 & 1.95 & 1.95 & 0.65 & 0.65 & 0.65 & 0.488 & 0.488 & & & 75.7 & 777 & 60000 \\
\hline 12 & 1.2 & 0.6 & 0.6 & 0.4 & 0.4 & 0.4 & 1.95 & 1.95 & 0.65 & 0.65 & 0.65 & 0.488 & 0.488 & 0.488 & & 90.9 & 949 & 82000 \\
\hline 13 & 1.2 & 0.6 & 0.6 & 0.4 & 0.4 & 0.4 & 1.95 & 1.95 & 0.65 & 0.65 & 0.65 & 0.488 & 0.488 & 0.488 & 0.488 & 91.4 & 1030 & 91000 \\
\hline
\end{tabular}
practically, concerning the connection of basis set variation and the associated property changes and in particular for hyperpolarizabilities. This information is of importance

TABLE IV. The effect of functions of various symmetries $(s, p, d, f)$ on the polarizabilities and hyperpolarizabilities of $\mathrm{NH}_{3} . "($ The property values are in a.u.)

- The geometry is from Ref. 47. The experimental and other computed values for the polarizability and hyperpolarizabilities are given in Table III. 


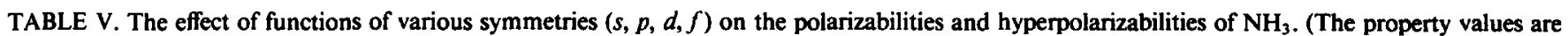
in a.u.)

\begin{tabular}{|c|c|c|c|c|c|c|c|c|c|c|c|c|c|c|c|c|c|c|}
\hline \multirow[b]{3}{*}{ No. } & \multicolumn{15}{|c|}{ Exponents } & \multirow[b]{3}{*}{$\alpha$} & \multirow[b]{3}{*}{$\beta$} & \multirow[b]{3}{*}{$\gamma$} \\
\hline & \multicolumn{6}{|c|}{$\mathrm{H}$} & \multicolumn{9}{|c|}{$\mathbf{N}$} & & & \\
\hline & $1 s$ & $2 s$ & $2 p$ & $3 s$ & $3 p$ & $3 d$ & $2 s$ & $2 p$ & $3 s$ & $3 p$ & $3 d$ & $4 s$ & $4 p$ & $4 d$ & $4 f$ & & & \\
\hline 1 & 1.2 & & & & & & 1.95 & 1.95 & & & & & & & & 2.6 & -1.6 & 11 \\
\hline 2 & 1.2 & 1.2 & & & & & 1.95 & 1.95 & & & & & & & & 7.5 & -12.8 & 27 \\
\hline 3 & 1.2 & 1.2 & 1.2 & & & & 1.95 & 1.95 & & & & & & & & 13.1 & -57.8 & 716 \\
\hline 4 & 1.2 & 1.2 & 1.2 & & & & 1.95 & 1.95 & 1.95 & & & & & & & 14.1 & 46.4 & 555 \\
\hline 5 & 1.2 & 1.2 & 1.2 & & & & 1.95 & 1.95 & 1.95 & 1.95 & & & & & & 15.3 & 37.9 & 410 \\
\hline 6 & 1.2 & 1.2 & 1.2 & & & & 1.95 & 1.95 & 1.95 & 1.95 & 1.95 & & & & & 22.5 & 0.6 & 118 \\
\hline 7 & 1.2 & 1.2 & 1.2 & 1.2 & & & 1.95 & 1.95 & 1.95 & 1.95 & 1.95 & & & & & 25.4 & 26.5 & 640 \\
\hline 8 & 1.2 & 1.2 & 1.2 & 1.2 & 1.2 & & 1.95 & 1.95 & 1.95 & 1.95 & 1.95 & & & & & 38.1 & 105 & 2500 \\
\hline 9 & 1.2 & 1.2 & 1.2 & 1.2 & 1.2 & 1.2 & 1.95 & 1.95 & 1.95 & 1.95 & 1.95 & & & & & 30.8 & 786 & 2200 \\
\hline 10 & 1.2 & 1.2 & 1.2 & 1.2 & 1.2 & 1.2 & 1.95 & 1.95 & 1.95 & 1.95 & 1.95 & 1.95 & & & & 32.2 & 61.3 & 2000 \\
\hline 11 & 1.2 & 1.2 & 1.2 & 1.2 & 1.2 & 1.2 & 1.95 & 1.95 & 1.95 & 1.95 & 1.95 & 1.95 & 1.95 & & & 37.3 & 34.1 & 1500 \\
\hline 12 & 1.2 & 1.2 & 1.2 & 1.2 & 1.2 & 1.2 & 1.95 & 1.95 & 1.95 & 1.95 & 1.95 & 1.95 & 1.95 & 1.95 & & 46.9 & -13.4 & 1300 \\
\hline 13 & 1.2 & 1.2 & 1.2 & 1.2 & 1.2 & 1.2 & 1.95 & 1.95 & 1.95 & 1.95 & 1.95 & 1.95 & 1.95 & 1.95 & 1.95 & 47.2 & 3.5 & 1700 \\
\hline 14 & 1.2 & 0.6 & 0.6 & & & & 1.95 & 1.95 & & & 1.3 & & & & & 12.8 & -45.5 & \\
\hline 15 & 0.9 & 0.45 & 0.45 & & & 0.3 & 1.95 & 1.95 & & & 1.3 & & & & 0.975 & & & 5240 \\
\hline
\end{tabular}

in the design of basis sets appropriate to satisfactorily describe electric properties. Thus, in order to study the behavior of the amine group orbitals [the orbitals for the ring have already been analyzed (Refs. 13 and 14)] we have chosen ammonia as a test model. The considered basis sets were systematically built and include up to $d$ orbitals for $\mathrm{H}$, and $f$ orbitals on $\mathrm{N}$, as required by perturbation theory for the evaluation of the second hyperpolarizability (Tables IV and V). The effect of varying the exponents on the properties, has also been considered (Tables VI-X).

We observe that addition of $2 s$ and $2 p$ functions on hydrogen, induces the following increase in the properties: $\alpha$, a factor (between 1 and 9 , usually); $\beta$, one order of magnitude; and $\gamma$, two orders of magnitude. This brings the computed results within the same order of magnitude as the experimentally determined ones. This finding shows how the standard CNDO method (only valence orbitals are used), which is inappropriate for quantitative predictions of hyperpolarizabilities, can be improved to a scheme capable of giving reasonably accurate values.

The results of Table $\mathrm{V}$ show that high 1 AOs like $f$ orbitals for nitrogen, and $d$ orbitals for hydrogen are sufficient (they can lead to reasonable results), but not necessary. This observation is in agreement with an analogous finding in hydrocarbons. ${ }^{13}$ Remarks concerning the redundancy of $f$ orbitals have also been made by Christiansen and McCullough. ${ }^{31}$ In general, it is noted that among two bases with the same symmetry functions, the higher properties ( $\alpha, \beta$, and $\gamma$ ) correspond to the basis set with the lower exponents, although some exceptions, in particular for the smaller bases, have been noted.

The above analysis and considerable computational experimentation in other molecules lead us to employ $2 s$ and $2 p$ for $\mathrm{N}$, and $1 s, 2 s$, and $2 p$ for $\mathrm{H}$.

In this section, we have confirmed the adequacy of our method (CHF-PT-EB-CNDO) and extended it by a chemically appealing and computationally economic approach for developing basis sets which allow a reasonable and balanced description of the polarized charge cloud.

\section{v. RESULTS AND DISCUSSION}

\section{A. Intramolecular processes in aniline}

Analysis of the changes in the electric properties, induced by the inversion of the amine group, shows that

TABLE VI. Effect of the variation of the nitrogen exponent on $\alpha, \beta$, and $\gamma$ of ammonia (in a.u.).

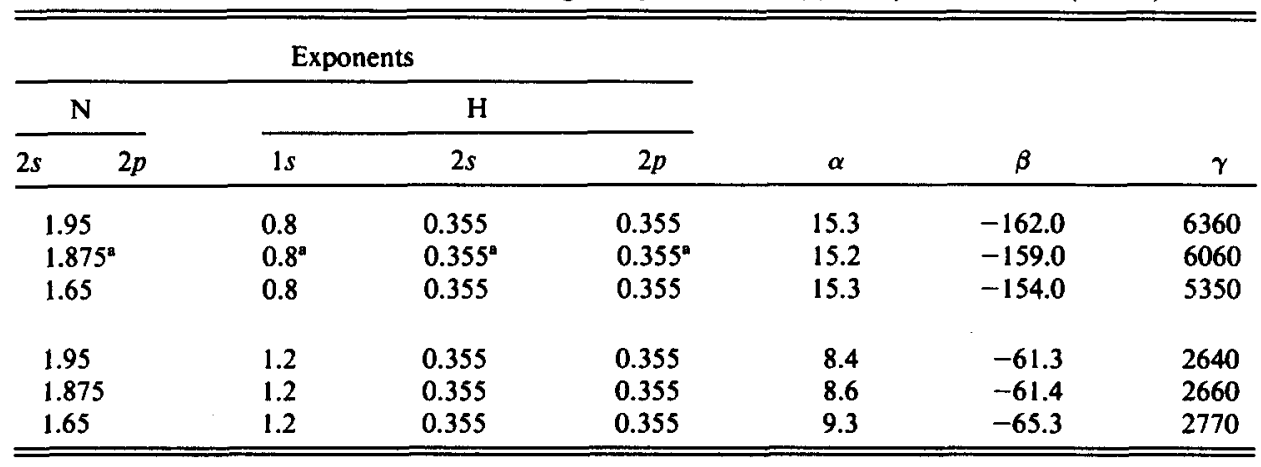

" These bases are used for subsequent computations of $\alpha$ and $\gamma$. Work in progress shows that these bases are adequate for the description of the electric properties in a series of amides and thus confirm their balanced nature. 
TABLE VII. Effect of the variation of the $1 s$ hydrogen exponent on $\alpha$, $\beta$, and $\gamma$ of ammonia (in a.u.).

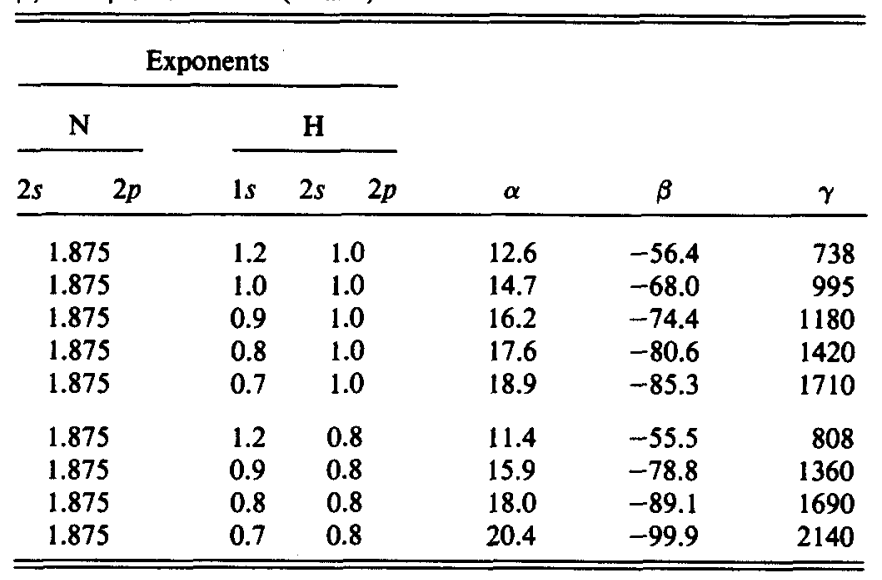

(Table XI): (a) The anisotropy values for $\alpha$ and $\gamma$ indicate that considerable charge rearrangement is associated with inversion of the amine group. It is noted that the anisotropies of $\alpha$ and $\gamma$ follow the same trend, that is for maximum coupling $\left(\varphi=0^{\circ}\right.$, Fig. 1), they reach their maximum value. It is observed that $\zeta^{2},|\Delta \alpha|$ and $|\Delta \gamma|$ present a minimum for the configuration which corresponds to the minimum energy. ${ }^{16}$ (b) The decoupling of the $\pi$ electron system and the charge reorganization through inversion, has a negligible effect on $\alpha$ and $\gamma$. (c) The first hyperpolarizability is a sensitive probe of the decoupling of the nitrogen lone pair from the ring $\pi$ electron system. It is observed that the value which corresponds to maximum coupling is (approximately) an order of magnitude larger than that which corresponds to minimal interaction.

Rotation of the amine group about the $\mathrm{N}-\mathrm{C}$ bond shows that (Table XII): (a) This process is also associated with a large charge rearrangement as the values of the anisotropies indicate. (b) The polarizability and second hyperpolarizability are rather insensitive to this process as they are to inversion. It is thought that the variation in $\alpha$ and $\gamma$, on the whole, may be rationalized by invoking the inductive effect which is associated with the atomic

TABLE VIII. Effect of the variation of the $2 s$ hydrogen exponent on $\alpha$, $\beta$, and $\gamma$ of ammonia (in a.u.).

\begin{tabular}{|c|c|c|c|c|c|c|}
\hline \multicolumn{4}{|c|}{ Exponents } & \multirow[b]{3}{*}{$\alpha$} & \multirow[b]{3}{*}{$\beta$} & \multirow[b]{3}{*}{$\gamma$} \\
\hline $\mathbf{N}$ & \multicolumn{3}{|c|}{$\mathbf{H}$} & & & \\
\hline $2 p$ & $1 s$ & $2 s$ & $2 p$ & & & \\
\hline 1.875 & 1.2 & 0.9 & 1.0 & 11.7 & -53.3 & 732 \\
\hline 1.875 & 1.2 & 0.8 & 1.0 & 10.9 & -49.7 & 703 \\
\hline 1.875 & 1.2 & 0.7 & 1.0 & 10.0 & -45.9 & 654 \\
\hline 1.875 & 1.2 & 0.6 & 1.0 & 9.3 & -42.2 & 590 \\
\hline 1.875 & 1.2 & 0.5 & 1.0 & 8.8 & -38.5 & 513 \\
\hline 1.875 & 1.2 & 0.4 & 1.0 & 8.4 & -34.7 & 419 \\
\hline 1.875 & 1.2 & 0.7 & 0.8 & 10.7 & -53.5 & 814 \\
\hline 1.875 & 1.2 & 0.6 & 0.8 & 10.1 & -51.4 & 799 \\
\hline 1.875 & 1.2 & 0.5 & 0.8 & 9.6 & -48.9 & 757 \\
\hline 1.875 & 1.2 & 0.4 & 0.8 & 9.2 & -45.1 & 659 \\
\hline 1.875 & 1.2 & 0.355 & 0.8 & 9.0 & -42.4 & 590 \\
\hline
\end{tabular}

TABLE IX. Effect of the variation of the $2 p$ hydrogen exponent on $\alpha, \beta$, and $\gamma$ of ammonia (in a.u.).

\begin{tabular}{|c|c|c|c|c|c|c|c|}
\hline \multicolumn{5}{|c|}{ Exponent } & \multirow[b]{3}{*}{$\alpha$} & \multirow[b]{3}{*}{$\beta$} & \multirow[b]{3}{*}{$\boldsymbol{\gamma}$} \\
\hline \multicolumn{2}{|c|}{$\mathbf{N}$} & \multicolumn{3}{|c|}{$\mathbf{H}$} & & & \\
\hline $2 s$ & $2 p$ & $1 s$ & $2 s$ & $2 p$ & & & \\
\hline & & 1.0 & 1.0 & 1.0 & 14.9 & -68.0 & 995 \\
\hline & & 1.0 & 1.0 & 0.7 & 15.6 & -71.1 & 954 \\
\hline & & 1.0 & 1.0 & 0.5 & 13.7 & -59.6 & 718 \\
\hline & & 1.0 & 1.0 & 0.4 & 11.9 & -48.1 & 533 \\
\hline & & 1.0 & 1.0 & 0.3 & 9.8 & -34.1 & 327 \\
\hline & & 1.0 & 1.0 & 0.2 & 8.0 & -21.2 & 149 \\
\hline & & 0.7 & 0.7 & 1.0 & 15.9 & -75.2 & 1590 \\
\hline & & 0.7 & 0.7 & 0.7 & 20.6 & -109 & 2450 \\
\hline & & 0.7 & 0.7 & 0.5 & 20.5 & -117 & 2690 \\
\hline & & 0.7 & 0.7 & 0.4 & 18.3 & -105 & 2410 \\
\hline & & 0.7 & 0.7 & 0.3 & 14.2 & -76 & 1670 \\
\hline & & 0.7 & 0.7 & 0.2 & 9.6 & -40 & 725 \\
\hline
\end{tabular}

electronegativities and thus has a less pronounced dependence on directional effects. ${ }^{32}$ (c) The first hyperpolarizability reflects, with great sensitivity, this process. The value which corresponds to maximum interaction is larger (approximately by a factor of 3 ) than that which corresponds to minimum coupling.

\section{B. Ammonia inversion}

The effect of the inversion of $\mathrm{NH}_{3}$ on its properties is now considered. This is a molecule where there is no charge transfer (CT) process, nor a coupled $\pi$ electron system and thus the effect of these factors on the magnitude of the properties of interest and their anisotropies may be elucidated. The results of Table XIII show: (a) As $\Theta$ (Fig. 2) increases, $\alpha, \beta$, and $\gamma$ decrease. (b) The variation of $\alpha$ with the inversion angle is very small and of $\gamma$ small (for example, the maximum and minimum values of $\gamma$ differ in percentage terms, employing the minimum value as reference point, by $16 \%$, which for most experimental

TABLE X. Effect of the variation of the hydrogen $2 s$ and $2 p$ exponents on $\alpha, \beta$, and $\gamma$ of ammonia (in a.u.).

\begin{tabular}{|c|c|c|c|c|c|c|c|}
\hline \multicolumn{5}{|c|}{ Exponents } & \multirow[b]{3}{*}{$\alpha$} & \multirow[b]{3}{*}{$\boldsymbol{\beta}$} & \multirow[b]{3}{*}{$\boldsymbol{\gamma}$} \\
\hline \multicolumn{2}{|c|}{$\mathbf{N}$} & \multicolumn{3}{|c|}{$\mathrm{H}$} & & & \\
\hline $2 s$ & $2 p$ & $1 s$ & $2 s$ & $2 p$ & & & \\
\hline & & 1.0 & & & 14.7 & -65.1 & 878 \\
\hline & & 1.0 & & & 14.9 & -68.0 & 995 \\
\hline & & 1.0 & & & 14.1 & -69.8 & 1120 \\
\hline & & 1.0 & & & 12.5 & -75.5 & 1420 \\
\hline & & 1.0 & & & 10.9 & -91.9 & 2800 \\
\hline & & 1.0 & & & 8.6 & -61.0 & 13400 \\
\hline & & 1.0 & & & 14.8 & -66.6 & 934 \\
\hline & & 1.0 & & & 14.9 & -69.7 & 1060 \\
\hline & & 1.0 & & & 14.2 & -71.4 & 1190 \\
\hline & & 1.0 & & & 12.6 & -76.6 & 1500 \\
\hline & & 1.0 & & & 10.9 & -91.9 & 2890 \\
\hline & & 1.0 & & & 8.4 & 75.5 & 12700 \\
\hline
\end{tabular}


TABLE XI. Effect of the inversion" of aniline on the polarizability", hyperpolarizability, and some of their anisotropies. ${ }^{b}$ The property values are presented in a.u.

\begin{tabular}{ccccccr}
\hline \hline & & & & \multicolumn{4}{c}{ Anisotropies } \\
\cline { 5 - 7 } $\begin{array}{c}\text { Angle } \\
(\varphi)^{c}\end{array}$ & $\alpha$ & $\beta$ & $\gamma$ & $\zeta^{2}$ & \multicolumn{1}{c}{$\Delta \alpha$} & \multicolumn{1}{c}{$\Delta \gamma$} \\
\hline $0.0^{\circ}$ & 77.9 & 185 & 38100 & 5540 & 37.6 & 27300 \\
$15.0^{\circ}$ & 77.9 & 174 & 38100 & 4620 & 30.9 & 23000 \\
$25.0^{\circ}$ & 77.8 & 157 & 38200 & 3400 & 20.3 & 16200 \\
$39.35^{\text {od }}$ & 77.7 & 124 & 38200 & 1910 & 0.5 & 3640 \\
$65.0^{\circ}$ & 77.3 & 42 & 38200 & 2390 & -45.3 & -25700 \\
$90.0^{\circ}$ & 77.3 & 15 & 38200 & 3520 & -58.3 & -34200 \\
\hline \hline
\end{tabular}

" The HNH angle remains constant at its experimental value (Ref. 16) The geometry is from Ref. 46.

b The ring orbitals, required for the computation of the properties, have been taken from (Ref. 43). For the amine group orbitals we have used as starting point the orbitals of Table II. These exponents have been readjusted a little in order to simulate as closely as possible the perturbed charge cloud of $\mathrm{C}_{6} \mathrm{H}_{5} \mathrm{NH}_{2}{ }^{\circ}$ Thus the following exponents, for the amine group, have been used: $\mathrm{N}: 2 s(1.875), 2 p(1.875), \mathrm{H}: 1 s(0.8342), 2 s(0.21)$, $2 p(0.21)$.

- The angle $\varphi$ is defined by the ring plane and the bisector of the HNH angle (Fig. 1).

This angle corresponds to the configuration which has the minimum energy in the inversion potential curve (Ref. 16).

- The experimental values are: $\alpha=77.8$ a.u. (Ref. 41 ), $\beta=153$ a.u. (Ref. 42), $\gamma=32600$ a.u. (Ref. 30) and 38900 a.u. (Ref. 42).

procedures is around the error margin). (c) The first hyperpolarizability is quite sensitive to changes in inversion angle.

It has been noted that the intramolecular processes considered in aniline induce large changes in $\beta$. It is worth investigating whether this large change is primarily due to the induced change in CT. The results of $\mathrm{NH}_{3}$ show that geometry changes induce large differences in the values of $\beta$. It is thus inferred that the primary cause for the sizable change in first hyperpolarizability values is the geometry variation and not CT effects.

\section{1-Aminonaphthalene}

The change in the electric property values of this molecule for three orientations are reported, to confirm or otherwise, their variation found in aniline. The results for $\beta$ should be considered as indicating a trend only, since there is little indication that the ring basis set is appropriate to determine the first hyperpolarizability. From the results of Table XIV we observe that: (a) The change in $\alpha$ is very small (it could be considered negligible) and in $\gamma$ is small. However there is a considerable change in $\beta$. Thus the minimum and the maximum values of $\beta$ differ approximately by a factor of 7 . (b) There is a pronounced sensitivity of the anisotropies and $\beta$ to geometry changes. The configuration which corresponds to maximum coupling and $\mathrm{CT}$ is associated with minimum

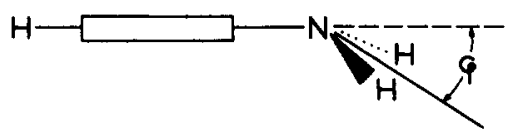

FIG. 1. Aniline inversion.
TABLE XII. Effect of the rotation of the amine group in aniline on its polarizability, hyperpolarizabilities, and some of their anisotropies." The property values are presented in a.u.

\begin{tabular}{lrrrrrrr}
\hline \hline & & & \multicolumn{3}{c}{ Anisotropies } \\
\cline { 5 - 8 } Angle & $\alpha$ & $\beta$ & $\gamma$ & $\gamma^{2}$ & $\Delta \alpha$ & $\Delta \gamma$ \\
\hline $0.0^{\circ b}$ & 77.6 & 41 & 39900 & 5040 & 38.9 & 34400 \\
$30.0^{\circ}$ & 77.6 & 65 & 39500 & 3120 & 25.4 & 23800 \\
$45.0^{\circ}$ & 77.6 & 87 & 39000 & 2310 & 14.9 & 15300 \\
$67.582^{\circ \mathrm{c}}$ & 77.7 & 114 & 38500 & 1940 & 3.9 & 6410 \\
$75.0^{\circ}$ & 77.7 & 119 & 38300 & 1920 & 2.0 & 4860 \\
$90.0^{\text {od }}$ & 77.7 & 124 & 38200 & 1910 & 0.5 & 3640 \\
\hline \hline
\end{tabular}

- The orbitals used for the computation of the properties are described in footnote $b$ of Table XI.

b The bisector of the HNH lies in the plane of the ring.

' One NH is eclipsed an ortho $\mathrm{H}$.

d This angle corresponds to the configuration with the minimum energy in the inversion potential curve (Ref. 16).

$|\beta|$. This further dissociates the first hyperpolarizability from the CT process. Also, if the magnitude of $\beta$ was primarily associated with $\mathrm{CT}$, then the configurations $c$ and $d$ (Table XIV) would have rather similar values for $\beta$; but this is not the case.

\section{The methyl derivatives of ammonia}

We have studied the effect of methyl substitution in $\mathrm{NH}_{3}$ on the electric properties. These model computations allow some insight to be gained into how $\mathrm{N}$ substitution of the hydrogens in a polyatomic molecule (e.g., aniline) affects the properties of the derived compound.

The results of Table XV show (a) the polarizability and $\beta$ uniformly increase with methylation. (b) The second hyperpolarizability has a minimum. (c) The first hyperpolarizability reflects, with remarkable sensitivity, the changes induced in the molecular structure by the process of methylation. Thus, for example, $\beta$ of $\mathrm{NH}_{3}$ and $\mathrm{N}\left(\mathrm{CH}_{3}\right)_{3}$ differs (approximately) by a factor of 30 .

TABLE XIII. The effect of the variation of the angle $\theta$ (Fig. 2) on the polarizability, hyperpolarizabilities, and the anisotropies" of $\mathrm{NH}_{3}$. The molecular quantities are in a.u.

\begin{tabular}{|c|c|c|c|c|c|c|}
\hline \multirow[b]{2}{*}{ Angle } & \multirow[b]{2}{*}{$\alpha$} & \multirow[b]{2}{*}{$\beta$} & \multirow[b]{2}{*}{$\gamma$} & \multicolumn{3}{|c|}{ Anisotropies } \\
\hline & & & & $\zeta^{2}$ & $\Delta \alpha$ & $\Delta \gamma$ \\
\hline $58^{\circ}$ & 15.7 & -61.5 & 6370 & 3.6 & -0.9 & 154 \\
\hline $63^{\circ}$ & 15.4 & -55.3 & 6210 & 7.4 & -2.7 & -270 \\
\hline $66.88^{\circ}$ & 15.3 & -49.9 & 6090 & 10.6 & -3.2 & -662 \\
\hline $67.88^{\circ}(\operatorname{expt})^{b}$ & 15.2 & -48.4 & 6060 & 11.4 & -3.4 & -770 \\
\hline $68.88^{\circ}$ & 15.2 & -46.8 & 6030 & 12.2 & -3.5 & -881 \\
\hline $73^{\circ}$ & 15.0 & -39.8 & 5890 & 15.0 & -3.9 & -1350 \\
\hline $78^{\circ}$ & 14.9 & -30.0 & 5720 & 17.6 & -4.2 & -1920 \\
\hline $84^{\circ}$ & 14.8 & -16.0 & 5550 & 19.2 & -4.4 & -2450 \\
\hline $90^{\circ}$ & 14.8 & 0.0 & 5490 & 19.7 & 2.2 & 1330 \\
\hline
\end{tabular}

- The computations for $\beta$ were performed by employing the following basis which was optimized with respect to $\mu$ and $\beta$ of $\mathrm{NH}_{3}: \mathrm{H}: 1 s(1.2)$, $2 s(0.64), 2 p(0.4562), \mathrm{N}: 2 s(1.875), 2 p(1.875)$; while $\alpha, \gamma$, and the anisotropies have been computed by employing the basis given in Table VI. It is noted that both the experimental and the computed value for $\mu$ is $1.5 \mathrm{D}$ (Ref. 40).

${ }^{b}$ The geometry is from Ref. 47. 


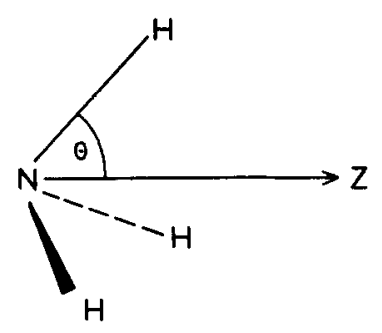

FIG. 2. Ammonia inversion.

It is noted that rotation of the methyl group [e.g., of $\mathrm{NH}\left(\mathrm{CH}_{3}\right)_{2}$ ] induces changes which lead to values that may differ by more than an order of magnitude and reverse the sign. ${ }^{48}$

\section{E. N,N-dimethylaniline}

It is interesting to note that substitution of two hydrogens by two methyl groups (Fig. 3) leads to a larger increase in $\alpha(36.2 \%)$ than $\gamma(25 \%)$, considering as a reference point the values for aniline.

From the results of Table XVI we observe, for the studied orientations: (a) The polarizability changes very little. The maximum $\alpha$, however, occurs when CNC (methyl carbons) is coplanar with the ring, similar to aniline. (b) The change in $\beta$ induced by the variation of geometry is pronounced. It is noted the coplanar configuration is not associated with maximum $|\beta|$. These results also indicate that geometry changes rather than the CT process are the predominant factors affecting $\beta$. (c) The change in $\gamma$ with the change in configuration is small like aniline. However, here there is a clearly defined maximum. (d) The anisotropy values sensitively reflect the changes in the charge cloud. Maximum values for the anisotropies are associated with the planar configuration similar to the results of aniline.

\section{CONCLUSIONS}

Support has been provided for the reliability of the method (CHF-PT-EB-CNDO) to allow a reasonable description of the polarized charge cloud. The present findings extend the applicability of CHF-PT-EB-CNDO

TABLE XIV. The properties $\alpha, \beta, \gamma, \zeta^{2}, \Delta \alpha$, and $\Delta \gamma$ (in a.u.) of 1-aminonaphthalene as a function of the molecular configuration.

\begin{tabular}{ccccccrr}
\hline \hline & & & & \multicolumn{3}{c}{ Anisotropies } \\
\cline { 5 - 8 } $\begin{array}{c}\text { Con- } \\
\text { figuration }\end{array}$ & $\alpha$ & $\beta$ & $\gamma$ & $\zeta^{2}$ & $\Delta \alpha$ & \multicolumn{1}{c}{$\Delta \gamma$} \\
\hline $\mathrm{c}$ & 127 & -28 & 84300 & 16000 & 37 & 37000 \\
$\mathrm{~d}$ & 127 & -114 & 86400 & 15400 & 35 & 39000 \\
$\mathrm{e}$ & 126 & -200 & 81800 & 12200 & -103 & -65700 \\
\hline \hline
\end{tabular}

- For the computation of the properties we have employed the ring orbitals proposed in Ref. 43 . While for the amine group the orbitals given in footnote $b$ of Table XI were used.

b The geometry of the rings (symmetric) and the $-\mathrm{NH}_{2}$ are from Refs. 14 and 46 , respectively.

c The ring and the amine group are coplanar.

d The $\mathrm{HNH}$ plane is perpendicular to the ring plane. The bisector of the HNH angle lies on the ring plane.

- The bisector of $\mathrm{HNH}$ angle is perpendicular to the ring plane.
TABLE XV. The polarizability and hyperpolarizabilities ${ }^{b}$ of ammonia and its methylated derivatives in a.u. ${ }^{a}$

\begin{tabular}{lccc}
\hline \hline Molecule $^{\mathrm{b}}$ & $\alpha$ & $\beta$ & \multicolumn{1}{c}{$\gamma$} \\
\hline $\mathrm{NH}_{3}$ & 15.2 & -30.0 & 6060 \\
$\mathrm{NH}_{2} \mathrm{CH}_{3}$ & 25.6 & -45.7 & 5950 \\
$\mathrm{NH}\left(\mathrm{CH}_{3}\right)_{2}^{\mathrm{c}}$ & 43.0 & -135 & 10500 \\
$\mathrm{~N}\left(\mathrm{CH}_{3}\right)_{3}$ & 64.7 & -880 & 18700 \\
\hline \hline
\end{tabular}

- For the amines $\mathrm{N}$ and $\mathrm{H}$ the basis defined in footnote a of Table XIII have been employed, while for the methyl group the orbitals presented in Ref. 12 have been used.

The geometries are from Ref. 47.

c One of the methyl hydrogens is eclipsed with the NH bond.

d Experimental and other theoretical results for $\mathrm{NH}_{3}$ are presented in Table III.
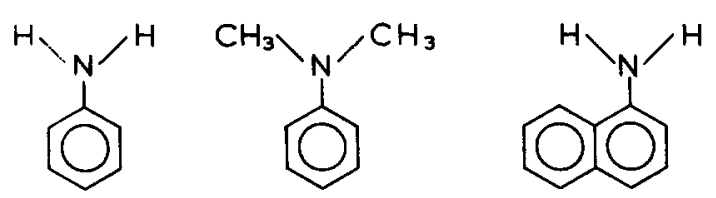

FIG. 3. Structure of the aromatic amines that were considered.

to $\mathrm{N}$ containing molecules. (Further, conclusive numerical evidence for the ability of CHF-PT-EB-CNDO to give reasonable results for nitrogen containing molecules is given in Ref. 29.

Detailed experimentation with many basis sets of various sizes (up to $f$ orbitals for $\mathrm{N}$, and $d$ orbitals for $\mathrm{H}$ have been considered) demonstrated once more ${ }^{12-14,29}$ the feasibility of using small, properly optimized bases to adequately describe polarization phenomena. We have shown that basis sets for complex molecules may be built from those of appropriately chosen fragments or models of them. These findings have been used to define a flexible and economic (in terms of computer time and storage) wave function, by which intramolecular processes in some amines have been analyzed.

The considered intramolecular processes (inversion and rotation) induce (a) small changes in $\alpha$ and $\gamma$ which

TABLE XVI. The properties $\alpha, \beta, \gamma, \zeta^{2}, \Delta \alpha$, and $\Delta \gamma$ (in a.u.) of $N-N$ dimethylaniline as a function of the molecular configuration.

\begin{tabular}{ccccccrr}
\hline \hline & & & & \multicolumn{4}{c}{ Anisotropies } \\
\cline { 5 - 8 } $\begin{array}{c}\text { Con- } \\
\text { figuration }\end{array}$ & $\alpha$ & $\beta$ & $\gamma$ & $\gamma^{2}$ & $\Delta \alpha$ & \multicolumn{1}{c}{$\Delta \gamma$} \\
\hline $\mathrm{c}$ & 135 & -340 & 44900 & 21600 & 136 & 60200 \\
$\mathrm{~d}$ & 132 & -450 & 45000 & 16400 & -103 & -47200 \\
$\mathrm{e}$ & 132 & -153 & 42800 & 15000 & 11 & 5580 \\
\hline \hline
\end{tabular}

- For the computation of the properties we have employed the ring orbitals given in Ref. 43; the nitrogen exponents given in the footnote b of Table $\mathrm{XI}$ and the methyl orbitals proposed in Ref. 12.

${ }^{b}$ The geometry of the ring and the $-\mathrm{N}\left(\mathrm{CH}_{3}\right)_{2}$ are from Refs. 46 and 47, respectively. The MeNMe is kept constant.

- The carbons of the two methyl groups lie in the ring plane.

d The bisector of the CNC (where the carbon atoms are from the methyl groups) lies in the ring plane and the CNC plane is perpendicular to the ring plane.

- The CNC (where the carbons are from the methyl groups) plane is perpendicular to the plane of the ring and the bisector of the CNC angle is perpendicular to the ring plane. 
TABLE XVII. Conversion of a.u. to electrostatic and SI units.

\begin{tabular}{lc}
\hline \hline Property & 1 a.u. equals (approx) \\
\hline$\alpha$ & $0.148176 \times 10^{-24}$ esu $\simeq 0.164867 \times 10^{-40} \mathrm{C}^{2} \mathrm{~m}^{2} \mathrm{~J}^{-1}$ \\
$\beta$ & $0.863993 \times 10^{-32} \mathrm{esu} \simeq 0.320662 \times 10^{-52} \mathrm{C}^{3} \mathrm{~m}^{3} \mathrm{~J}^{-2}$ \\
$\gamma$ & $0.503717 \times 10^{-39} \mathrm{esu} \simeq 0.623597 \times 10^{-64} \mathrm{C}^{4} \mathrm{~m}^{4} \mathrm{~J}^{-3}$ \\
\hline
\end{tabular}

may be interpreted by the inductive effect; (b) large changes in the anisotropies of the charge cloud; and (c) large changes in $\beta$.

The relation of the decoupling of the $\pi$ electron system to $\beta$ is rather intriguing. It is clear that decoupling and change of $\beta$ share a common cause; the geometry change. It is not considered, however, likely that reduction of CT will, in general, lead to a reduction in $\beta$. This view is supported by the results of $\mathrm{C}_{6} \mathrm{H}_{5} \mathrm{NH}_{2}, \mathrm{C}_{6} \mathrm{H}_{5} \mathrm{~N}\left(\mathrm{CH}_{3}\right)_{2}$, and $\mathrm{C}_{10} \mathrm{H}_{7} \mathrm{NH}_{2}$. Conversion factors of a.u. to other units are given in Table XVII.

In summary, it is noted that the considered intramolecular processes have a pronounced effect on the directional properties (e.g., anisotropies, $\beta$ ) of the charge cloud.

${ }^{1}$ G. R. Meredith and B. Buchalter, J. Chem. Phys. 78, 1615 (1983); G. R. Meredith, B. Buchalter, and C. Hanzlik, ibid. 78, 1533, 1543 (1983); B. F. Levine and C. G. Bethea, ibid. 66, 1070 (1977); 65, 2439 (1976).

${ }^{2}$ R. M. Berns and P. E. S. Wormer, Mol. Phys. 44, 1215 (1981).

${ }^{3}$ G. H. F. Diercksen, V. Kellö, and A. J. Sadlej, J. Chem. Phys. 79, 2918 (1983); G. D. Purvis III and R. J. Bartlett, Phys. Rev. 23, 1594 (1981); O. Zamani-Khamiri, E. F. McIntyre, and H. F. Hameka, J. Chem. Phys. 72, 1280, 5906 (1980).

4 M. P. Bogaard, A. D. Buckingham, and G. L. D. Ritchie, J. Chem. Soc. Faraday Trans. 2 77, 1547 (1981).

5 J. P. Hermann, Opt. Commun. 9, 74 (1973).

${ }^{6}$ R. J. Bartlett and G. D. Purvis III, Phys. Rev. A 20, 1313 (1979).

${ }^{7}$ R. J. Bartlett and G. D. Purvis III, Phys. Scr. 21, 255 (1980).

${ }^{8}$ R. D. Amos and J. H. Williams, Chem. Phys. Lett. 84, 104 (1981).

${ }^{9}$ G. R. Meredith and B. Buchalter, J. Chem. Phys. 78, 1938 (1983).

${ }^{10}$ B. F. Levine, C. G. Bethea, E. Wasserman, and L. Leenders, J. Chem. Phys. 68, 5042 (1978).

"J. Maddox, Nature 306, 311 (1983).

${ }^{12}$ C. A. Nicolaides, M. G. Papadopoulos, and J. Waite, Theor. Chim. Acta 61, 427 (1982).

${ }^{13}$ M. G. Papadopoulos, J. Waite, and C. A. Nicolaides, J. Chem. Phys. 77, 2527 (1982).
14 J. Waite, M. G. Papadopoulos, and C. A. Nicolaides, J. Chem. Phys. 77, 2536 (1982).

${ }^{15}$ A. Wolf, U. Voets, and H. H. Schmidtke, Theor. Chim. Acta 54, 229 (1980).

${ }^{16}$ C. C. Strametz and H. H. Schmidtke, Theor. Chim. Acta 42, 13 (1976).

${ }^{17}$ W. J. Hehre, L. Radom, and J. A. Pople, J. Am. Chem. Soc. 94, 1496 (1972).

${ }^{18}$ E. F. Mclntyre and H. F. Hameka, J. Chem. Phys. 70, 2215 (1979).

19 J. Zyss, J. Chem. Phys. 70, 3333 (1979).

${ }^{20}$ J. Zyss, J. Chem. Phys. 70, 3341 (1979).

${ }^{21}$ J. Zyss, J. Chem. Phys. 71, 909 (1979).

${ }^{22}$ J. Zyss and G. Berthier, J. Chem. Phys. 77, 3635 (1982).

${ }^{23}$ S. T. Lalama and A. F. Garito, Phys. Rev. A 20, 1179 (1979).

${ }^{24}$ B. F. Levine and C. G. Bethea, J. Chem. Phys. 65, 2429 (1976); K. D. Singer and A. F. Garito, ibid. 75, 3572 (1981).

${ }^{25}$ I. Ledoux and J. Zyss, Chem. Phys. 73, 203 (1982).

${ }^{26}$ A. D. Buckingham and B. J. Orr, Q. Rev. (London) 21, 195 (1967).

${ }^{27}$ A. K. Burnham, L. W. Buxton, and W. H. Flygare, J. Chem. Phys. 67, 4990 (1977).

${ }^{28}$ R. McWeeny, Phys. Rev. 126, 1028 (1962); G. Diercksen and R. McWeeny, J. Chem. Phys. 44, 3554 (1966); J. L. Dodds, R. McWeeny, W. T. Raynes, and J. P. Riley, Mol. Phys. 33, 611 (1977).

${ }^{29}$ J. Waite and M. G. Papadopoulos, J. Chem. Phys. (submitted).

${ }^{30}$ J. L. Oudar, D. S. Chemla, and E. Batifol, J. Chem. Phys. 67, 1626 (1977).

${ }^{31}$ P. A. Christriansen and E. A. McCullough Jr., Chem. Phys. Lett. 63, 570 (1979).

${ }^{32}$ J. N. Murrell, S. F. A. Kettle, and J. M. Tedder, The Chemical Bond (Wiley, New York, 1978).

${ }^{33}$ A. J. Sadlej, Mol. Phys. 34, 731 (1977).

${ }^{34} \mathrm{H}$. Shinoda and T. Akutagawa, Bull. Chem. Soc. Jpn. 48, 731 (1977).

${ }^{35}$ E. N. Svendsen and T. Stroyer-Hansen, Int. J. Quantum Chem. 13, 235 (1978).

${ }^{36}$ J. J. C. Teixeira-Dias and P. J. Sarre, J. Chem. Soc. Faraday Trans. 2 71, 906 (1975).

${ }^{37}$ N. J. Bridge and A. D. Buckingham, Proc. R. Soc. London Ser. A 295, 334 (1966).

${ }^{38}$ N. S. Hush and M. L. Williams, Theor. Chim. Acta 25, 346 (1972).

${ }^{39}$ P. Lazzeretti and R. Zanasi, J. Chem. Phys. 74, 5216 (1981).

${ }^{40}$ J. F. Ward and C. K. Miller, Phys. Rev. A 19, 826 (1979).

${ }^{41}$ M. J. Aroney and R. J. W. LeFévre, J. Chem. Soc. 1960, 2161.

42 J. L. Oudar and D. S. Chemla, J. Chem. Phys. 66, 2664 (1977).

${ }^{43} \mathrm{~J}$. Waite and M. G. Papadopoulos, J. Comp. Chem. 4, 578 (1983).

44 G. Burns, J. Chem. Phys. 41, 1521 (1964).

${ }^{45} \mathrm{~J}$. A. Pople and D. L. Beveridge, Approximate Molecular Orbital Theory (McGraw-Hill, New York, 1970).

${ }^{46}$ D. G. Lister and J. K. Tyler, Chem. Commun. 1966, 152.

${ }^{47}$ M. J. S. Dewar and W. Thiel, J. Am. Chem. Soc. 99, 4907 (1977).

48 J. Waite and M. G. Papadopoulos (unpublished).

${ }^{49}$ C. Moore, Atomic Energy Levels, Natl. Bur. Stand. U.S. Circ. No. 467 (U.S. GPO, Washington, D.C., 1949). 